\title{
Short-term outcome of laparoscopic cholecystectomy for benign gall bladder diseases in 76 dogs
}

\author{
Hiroo KANAI ${ }^{1,6)}$, Ken HAGIWARA ${ }^{2,6) *}$, Aya NUKAYA ${ }^{3,6)}$, Motoki KONDO ${ }^{4,6)}$ and \\ Toshihide ASO ${ }^{5,6)}$ \\ ${ }^{1)}$ Kanai Veterinary Surgery, 569-1 Nozato, Himeji-shi, Hyogo 670-0811, Japan \\ ${ }^{2)}$ Department of Digestive Surgery, Nihon University School of Medicine, 1-6 Kandasurugadai, Chiyoda-ku, \\ Tokyo 101-8309, Japan \\ ${ }^{3)}$ Aya Animal Hospital, 4-1-17 Fujinokidai, Nara-shi, Nara 631-0044, Japan \\ ${ }^{4)}$ Apple Tree Animal Hospital, 52-2 Hiruta, Takatanacho, Anjo-shi, Aichi 446-0053, Japan \\ ${ }^{5)}$ Animal Medical Center ALOHA, 5-12-5 Hikino, Fukuyama-shi, Hiroshima 721-0942, Japan \\ ${ }^{6)}$ Study Group of Small Animal Minimal Invasive Treatment (SAMIT), 569-1 Nozato, Himeji-shi, Hyogo 670-0811, \\ Japan
}

J. Vet. Med. Sci.

80(11): 1747-1753, 2018

doi: 10.1292/jvms.18-0266

Received: 12 May 2018

Accepted: 27 August 2018

Published online in J-STAGE:

20 September 2018
ABSTRACT. Laparoscopic cholecystectomy $(\mathrm{LC})$ is widely accepted as the standard treatment for benign gall bladder diseases in humans because it has proven to be less invasive and safer than are traditional methods. However, the efficacy of LC in dogs remains unclear. The present study aimed to examine the short-term outcome of LC for benign gall bladder diseases in dogs. We enrolled 76 consecutive dogs that underwent LC for benign gall bladder diseases at our hospital between April 2008 and October 2016. Dogs with jaundice, gall bladder ruptures, abdominal effusion, or extrahepatic biliary obstruction were not excluded from the indication. Factors including age, body weight, sex, clinical sign, disease, operative time, conversion to open surgery, perioperative complications, and postoperative hospital stay were investigated. The median age of the dogs was 11 years, and the median body weight was $5.4 \mathrm{~kg}$. Fifty percent of the dogs exhibited no symptoms at the initial visit. Preoperative elevation of total bilirubin levels was observed in 16 dogs (21\%). LC was successfully completed in 71 dogs (93\%); the median operative time was $124 \mathrm{~min}$. Although gall bladder ruptures were observed in $2(2.6 \%)$ dogs, the operations were completed successfully. Three dogs $(4.1 \%)$ had to be converted to open cholecystectomy and $2(2.6 \%)$ underwent reoperation. Two dogs (2.6\%) died intraoperatively and $2(2.6 \%)$ died postoperatively. LC was a feasible, safe, and appropriate procedure considering the current operative indications for benign gall bladder diseases in dogs.

KEY WORDS: benign gall bladder disease, dog, laparoscopic cholecystectomy

Benign gall bladder diseases, such as gall bladder mucocele, cholecystitis, and cholelithiasis, are common in dogs. A standard treatment for benign gall bladder diseases in dogs is open cholecystectomy. In contrast, laparoscopic cholecystectomy has been widely accepted as the standard treatment in humans because it has proven to be both less invasive and safer than are traditional methods $[4,9,15]$.

In dogs, laparoscopic surgery has recently become widely used, and is highly regarded over open surgery because of its reduced invasiveness $[3,5,7,13]$. Furthermore, the advent of magnification during operations has led to increased anatomical understanding. Therefore, laparoscopic surgery in dogs could possibly become an even more effective treatment for benign gall bladder diseases and other conditions.

Although some reports have described laparoscopic cholecystectomy in dogs [12, 17], its efficacy remains unclear because of the small number of cases reported in previous studies. Moreover, the indications for laparoscopic cholecystectomy were rigorously limited.

We began to perform laparoscopic cholecystectomy for benign gall bladder diseases in dogs in 2008. Since then, we have accumulated experience in many cases. The present study aimed to examine the short-term outcome of laparoscopic cholecystectomy for benign gall bladder diseases in dogs. 

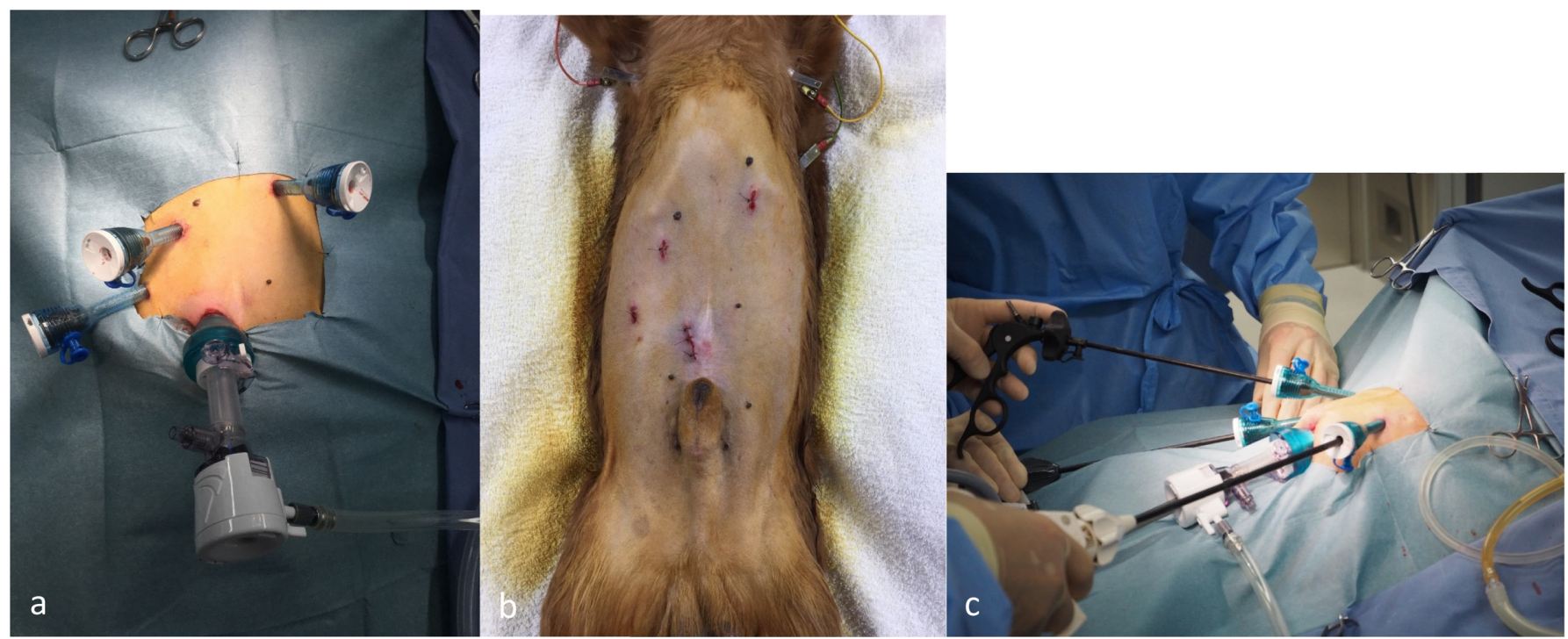

Fig. 1. a, b: Trocar placement. c: Operative view.

\section{MATERIALS AND METHODS}

Dogs that underwent laparoscopic cholecystectomy for benign gall bladder diseases consecutively at Kanai Veterinary Surgery between April 2008 and October 2016 were enrolled in the present study. We obtained informed consent from all the owners of the dogs before conducting the operation. Benign gall bladder diseases, such as gall bladder mucocele, cholecystitis, and cholelithiasis, were defined as indications. We preoperatively diagnosed each disease by using abdominal ultrasonography, and then confirmed the diagnoses by using pathological examinations of resected specimens, culture tests, and calculus analyses. Dogs with jaundice, evidence of gall bladder rupture, abdominal effusion, or extrahepatic biliary obstruction were not excluded from the indication of laparoscopic cholecystectomy in the present study. Complete blood counts, serum biochemistry analyses including $\gamma$-glutamyltransferase level, ultrasonography, and thoracic radiography were routinely performed in all dogs prior to operation. Computed tomography was not performed routinely and was used depending on the condition of each dog.

The method of laparoscopic cholecystectomy was as follows. Under general anesthesia, the dogs were positioned in dorsal recumbency. Laparoscopic cholecystectomy was performed using a 4-trocar technique. The trocar placement used in our procedure has been shown in Fig. 1. Abdominal access was obtained using the Hasson technique, and a 10-mm blunt trocar was inserted into the abdomen. The abdomen was insufflated with $\mathrm{CO}_{2}$ to a maximum of $8-12 \mathrm{mmHg}$ by using a regulatory mechanical insufflator. Three other trocars were inserted under laparoscopic guidance. The first trocar $(12 \mathrm{~mm})$ was placed in the left cranial quadrant, the second $(5 \mathrm{~mm})$ in the right cranial quadrant, and the third $(5 \mathrm{~mm})$ in the right middle portion of the abdomen. A $30^{\circ}$ laparoscope (KARL STORZ SE \& Co. KG, Tuttlingen, Germany) was used. We initially used an electric scalpel. However, recently we have started routinely using an ultrasonic incision device for dissecting the gall bladder from the fossa. The cystic duct was ligated using 10-mm metallic clips in the initial 36 cases and a 3-0 VICRYL ${ }^{\circledR}$ suture (Ethicon, Inc., Somerville, NJ, U.S.A.) or a combination of these methods in the subsequent cases. We usually used atraumatic forceps. However, we sometimes encountered cases in which we could not grasp the gall bladder wall (this occurred in dogs with both mucocele and cholecystitis). Simultaneously, we achieved an appropriate operative view by pushing up the gall bladder by using either forceps or surgical sponge and gauze grasped with forceps.

\section{Conventional procedure}

Between 2008 and 2012, we elevated the gall bladder by using grasp forceps through the right middle trocar, and visualized Calot's triangle (Fig. 2). We always dissected and transected the cystic duct prior to dissecting the gall bladder free from the hepatic fossa. Subsequently, we performed a retrograde dissection of the gall bladder.

\section{Current procedure}

Since 2012, we have been dissecting and securing the cystic

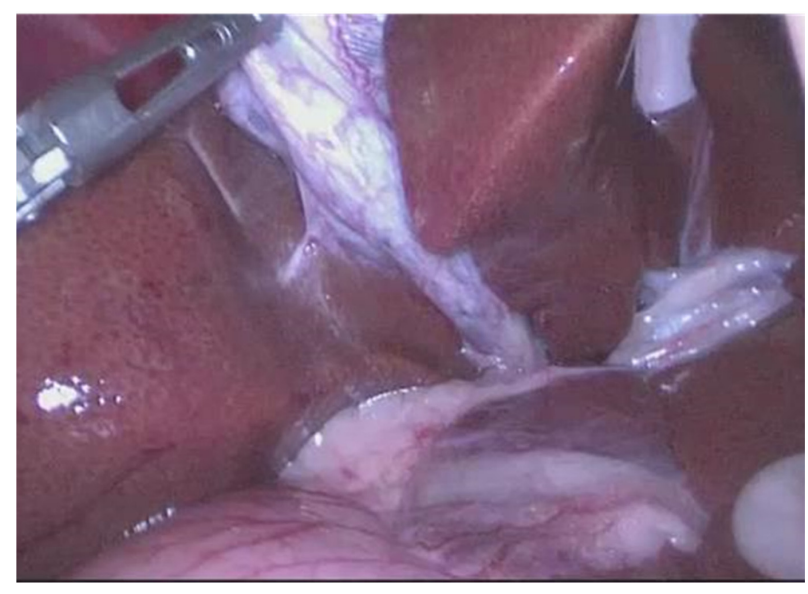

Fig. 2. Visualization of Calot's triangle. 

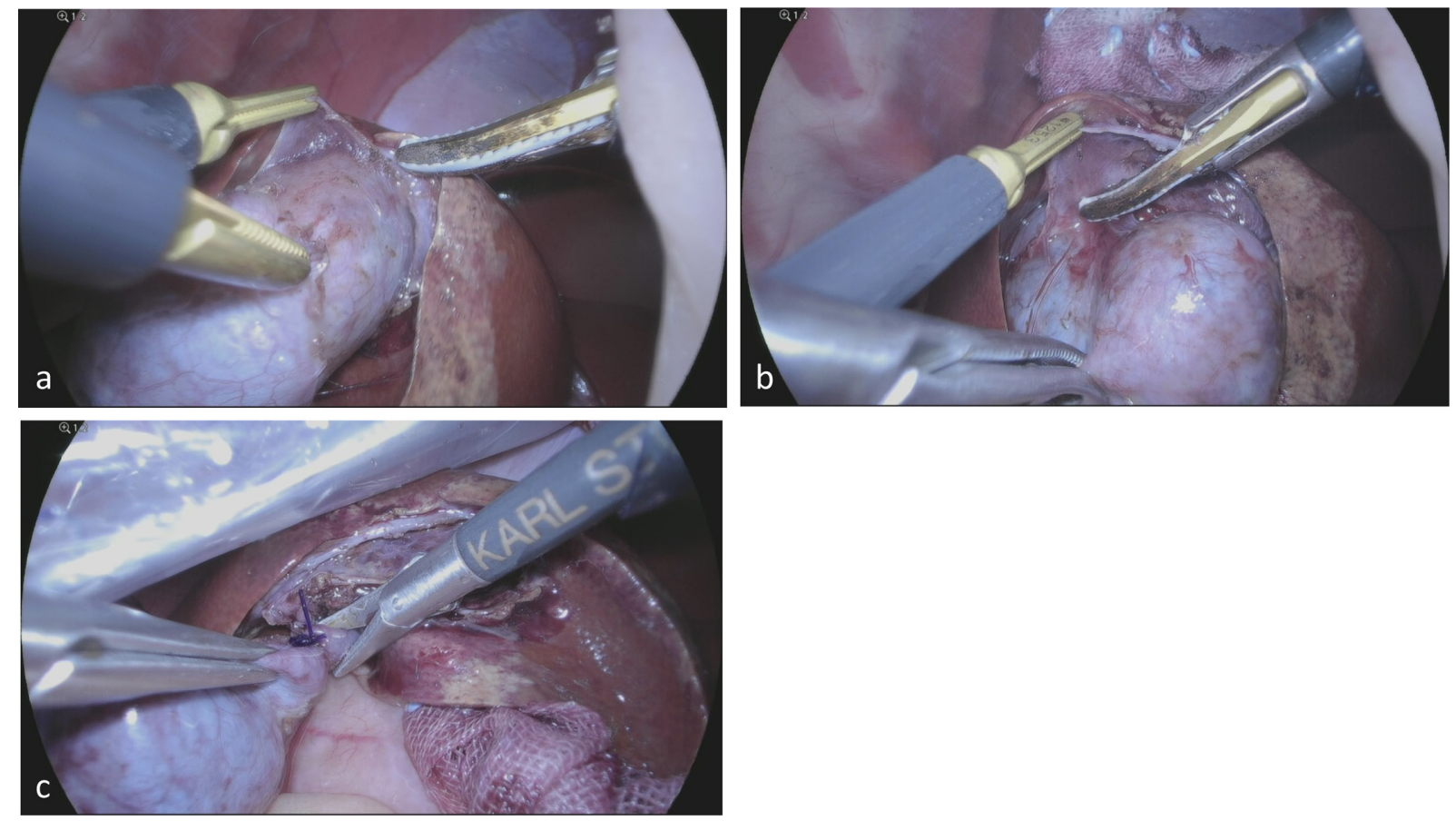

Fig. 3. a, b: Dissection of the gall bladder from the hepatic fossa along the subserosal layer. c: Transection of the cystic duct by using Metzenbaum scissors after a ligation.

duct after visualizing Calot's triangle, but we do not transect it at this point. We dissected the gall bladder from the hepatic fossa prior to transection of the cystic duct anterogradely. Subsequently, we ligated the cystic duct and transected it using laparoscopic Metzenbaum scissors (Fig. 3). In the case of a high probability of common bile duct injury or bleeding from the liver resulting from the dissection of the cystic duct, we dissected the gall bladder from the hepatic fossa initially without attempting to do so by force.

\section{Intraoperative cholangiography}

We have been performing intraoperative cholangiography to confirm the status of the common bile duct as a routine procedure since 2008. After transecting the cystic duct, a catheter was inserted through the stump of the cystic duct into the common bile duct (Fig. 4). A 4- to 7-Fr feeding tube (Atom Medical Corp., Saitama, Japan), whose tip was blunt, was used as a catheter. Approximately 3 to $5 \mathrm{~m} l$ of iodixanol was injected slowly. However, we did not perform it in cases that had little chance of the gall bladder content being obstructed by debris and sludge in the common bile duct. After confirming the patency of the common bile duct, we removed the catheter and ligated the cystic duct.

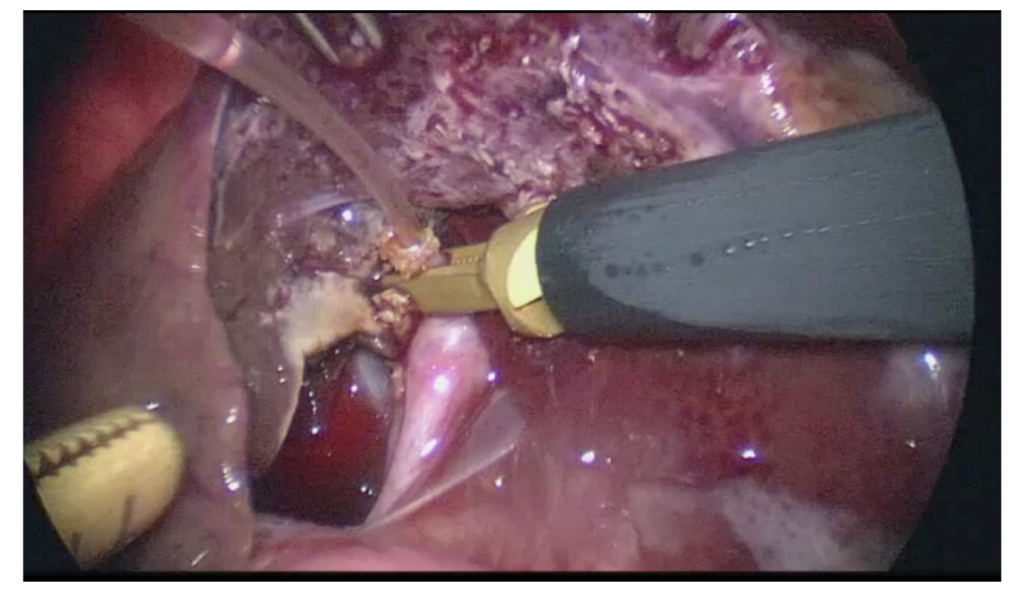

Fig. 4. Catheter insertion through the stump of the cystic duct for cholangiography. 
Table 1. Breed of dogs

\begin{tabular}{lc}
\hline \multicolumn{1}{c}{ Breed } & No. of dogs \\
\hline Miniature Dachshunds & 25 \\
Toy Poodle & 10 \\
Miniature Schnauzer & 9 \\
Chihuahua & 4 \\
Pomeranian & 4 \\
Shih Tzu & 3 \\
Papillion & 3 \\
American Cocker Spaniel & 3 \\
Shetland Sheepdog & 3 \\
Japanese Shiba inu & 2 \\
Yorkshire Terrier & 2 \\
Mixed breed & 2 \\
Maltese dog & 1 \\
Pug & 1 \\
Bernese Mountain Dog & 1 \\
French Bulldog & 1 \\
Border Collie & 1 \\
Wire Fox Terrier & 1 \\
\hline
\end{tabular}

Table 2. Characteristics of the dogs

\begin{tabular}{lc}
\hline \multicolumn{2}{c}{ Variable } \\
\hline Sex \\
$\quad$ Male & 34 \\
$\quad$ Female & 41 \\
Age (years) & \\
$\quad$ Mean (SD) & $10.3(3.1)$ \\
$\quad$ Median (range) & $11(1-16)$ \\
Weight (kg) & \\
$\quad$ Mean (SD) & $6.8( \pm 5.7)$ \\
$\quad$ Median (range) & $5.4(1.2-41.7)$ \\
Castrated or Spayed & 48 \\
Indication & \\
$\quad$ Cholecystolithiasis & 16 \\
$\quad$ Cholecystitis & 23 \\
$\quad$ Mucocele & 37 \\
Abdominal effusion & 2 \\
Ruptured gall bladder & 2 \\
Icterus & 16 \\
\hline
\end{tabular}

Table 3. Clinical signs at the initial visit

\begin{tabular}{lc}
\hline Clinical signs & $\begin{array}{c}\text { No. of dogs (\%) } \\
\text { (multiple answers possible) }\end{array}$ \\
\hline Appetite loss & $22(29 \%)$ \\
Icterus & $16(21 \%)$ \\
Abdominal pain & $9(12 \%)$ \\
Vomiting & $9(12 \%)$ \\
Lethargy & $7(9.2 \%)$ \\
Diarrhea & $2(2.6 \%)$ \\
None & $38(50 \%)$ \\
\hline
\end{tabular}

Liver biopsy was performed in most of the dogs. The gall bladder was retrieved through the subumbilical port site incision. In some dogs, a 19-Fr drain tube was placed around the liver fossa and extracted after confirming the condition of drainage.

One experienced veterinarian performed laparoscopic cholecystectomy in all dogs.

Characteristic indices (age, body weight, sex, breed, clinical sign, and disease) and operative indices (intraoperative finding, operative time, conversion to open surgery, drain placement, perioperative complications, and postoperative hospital stay) were investigated. A grade 3 or higher operative complication, in accordance with the Clavien-Dindo classification, was defined as an operative complication in the present study [8]. Death within 1 week of the operation was defined as perioperative death. Data were retrieved from the medical records of all dogs.

\section{RESULTS}

In total, 76 dogs were analyzed consecutively in the present study. Table 1 shows the breeds of the 76 dogs that underwent laparoscopic cholecystectomy. Miniature Dachshunds outnumbered the other dog breeds. The characteristics are summarized in Table 2. The median age of the dogs was 11 years (range: $1-16$ years), and the median body weight was $5.4 \mathrm{~kg}$ (range: 1.2-41.7). There were 34 male and 41 female dogs (sex was not listed for $1 \mathrm{dog}$ ). Table 3 shows the clinical symptoms of all the dogs; $50 \%$ of the dogs showed no symptoms at their first hospital visit.

Table 4 shows the perioperative outcomes of the dogs that underwent laparoscopic cholecystectomy. Laparoscopic cholecystectomy was successfully completed in 71 dogs (93\%). The median operative time was 124 min (range: 55-210 min.), except for 5 dogs ( 3 of these had to be converted to open surgery and the other 2 died intraoperatively). The median operative time for the last $20 \mathrm{dogs}$ in the present study was $112.5 \mathrm{~min}$. Combined surgery was performed in 7 dogs $(9.2 \%)$. We performed ovariohysterectomy in 3 dogs, hepatectomy in 2, cystotomy in 1, and fenestration of a liver cyst in 1 . Preoperative elevation of total bilirubin concentration was observed in 16 dogs (21\%). Intraoperative abdominal effusion was observed in 2 dogs (2.6\%). We encountered gall bladder ruptures in $18 \mathrm{dogs}(24 \%)$; the gall bladder was already ruptured in 2 dogs $(2.6 \%)$, and it ruptured during surgery in $16(21 \%)$. However, we successfully completed the operation after suctioning the contents of the gall bladder and irrigating the abdominal cavities in these dogs. A drain tube was placed in 19 dogs $(25 \%)$. Cholangiography was performed to confirm the patency of the common bile duct in $57 \mathrm{dogs}(75 \%)$. Three dogs $(4.1 \%)$ had to be converted to open cholecystectomy, in addition to the 2 dogs that died intraoperatively (Table 5). This was due to difficulty in maintaining respiratory conditions under pneumoperitoneum in $1 \mathrm{dog}$ and severe intraabdominal adhesion in 2. The median postoperative hospital stay was 3 days (range: 1-23 days).

Table 6 shows that intraoperative death occurred in 2 dogs. One dog died because of acute circulatory failure and the other because of an injury to the diaphragm caused by the electric scalpel. Two dogs also died within 1 week of the operation. One dog died because of liver dysfunction 5 days after the operation. The other dog died because of sudden acute heart failure 2 days after the operation. Two dogs underwent reoperation because of bile peritonitis caused by an injury to the remnant cystic duct. One dog underwent reoperation 3 days after the initial operation and the other underwent reoperation 4 days after the initial operation. Neither of these dogs had any intraoperative complications during the initial operation. These dogs recovered without further complications and were discharged from the hospital. 
Table 4. Perioperative outcomes of laparoscopic cholecystectomy

\begin{tabular}{lc}
\hline Variable & \\
\hline Operative time (min) & $124( \pm 34)$ \\
$\quad$ Mean (SD) & $121(55-210)$ \\
$\quad$ Median (range) & $7(9.2 \%)$ \\
Combined operation & $3(4.1 \%)$ \\
$\quad$ Ovariohysterectomy & $2(2.6 \%)$ \\
$\quad$ Hepatectomy & $1(1.3 \%)$ \\
Cystotomy & $1(1.3 \%)$ \\
Fenestration of liver cyst & $19(25 \%)$ \\
Placement of drain & $57(75 \%)$ \\
Cholangiography & $3(4.1 \%)$ \\
Conversion to open surgery & \\
Length of hospital stay (day) & \\
$\quad$ Mean (SD) & $5.4( \pm 4.6)$ \\
$\quad$ Median (range) & $3.5(1-23)$ \\
Complication & $9(11.8 \%)$ \\
Reoperation & $2(2.6 \%)$ \\
In-hospital mortality & $4(5.3 \%)$ \\
$\quad$ Intraoperative & $2(2.6 \%)$ \\
$\quad$ Postoperative & $2(2.6 \%)$ \\
\hline
\end{tabular}

Table 5. Summary of cases of conversion to open cholecystectomy

\begin{tabular}{|c|c|c|c|c|c|c|}
\hline Breed & Sex & $\begin{array}{c}\text { Age } \\
\text { (years) }\end{array}$ & $\begin{array}{l}\text { BW } \\
(\mathrm{kg})\end{array}$ & Disease & Date & Cause \\
\hline Pomeranian & Female & 14 & 5.1 & Cholecystitis & 2012 & Severe adhesion \\
\hline Miniature Dachshunds & Female & 13 & 6.9 & Cholecystitis & 2015 & Severe adhesion \\
\hline American Cocker Spaniel & Female & 13 & 10 & Mucocele & 2015 & $\begin{array}{l}\text { Deterioration of } \\
\text { respiratory condition }\end{array}$ \\
\hline
\end{tabular}

Table 6. Summary of cases of intraoperative death

\begin{tabular}{llccccl}
\hline \multicolumn{1}{c}{ Breed } & Sex & $\begin{array}{c}\text { Age } \\
(\text { years })\end{array}$ & $\begin{array}{c}\text { BW } \\
(\mathrm{kg})\end{array}$ & Disease & Date & \multicolumn{1}{c}{ Cause } \\
\hline Miniature Schnauzer & Female & 10 & 8.1 & Mucocele & 2009 & Acute circulatory failure \\
Miniature Dachshunds & Male & 11 & 5.3 & Mucocele & 2011 & Injury of diaphragm \\
\hline
\end{tabular}

\section{DISCUSSION}

This study investigated the feasibility of laparoscopic cholecystectomy for benign gall bladder diseases in dogs. We showed that laparoscopic cholecystectomy could be completed safely and could have similar advantages in dogs as in humans, such as lowered invasiveness and good cosmetic outcome. Therefore, it may be used as a standard therapy in most cases of canine benign gall bladder diseases. Previous studies have shown that laparoscopic surgery is one of the most effective treatments for various diseases in dogs $[3,5,7,13]$. Laparoscopic surgery for female sterilization has already been widely accepted. However, only a few reports regarding laparoscopic cholecystectomy in dogs have been published to date $[12,17]$. The reasons for this are as follows. First, previous studies have shown that a gall bladder disease, in particular, a gall bladder mucocele or acute cholecystitis, has a high mortality rate because of a high incidence of ruptures and bile peritonitis during operations $[2,6,11,14,16,20]$. Second, advanced skills are required to perform laparoscopic cholecystectomy because of the anatomical variations in the connection between the cystic duct and common bile duct; moreover, adhesion between the gall bladder and liver fossa is frequently observed. Further, the gall bladder in the case of mucocele and cholecystitis is often swollen, which worsens the visualization of the operative field. In addition, the gall bladder wall is fragile, and thus, likely to rupture in the case of gall bladder mucocele and acute cholecystitis. Inserting a catheter through the remnant of the cystic duct is also difficult because of cholangiography being performed in cases of a suspected obstruction of the common bile duct.

Mayhew et al. reported excellent results of laparoscopic cholecystectomy for gall bladder mucocele in 6 dogs [12]. It was reported that 2 cases with clinical sign of biliary tract rupture were had to be converted to open cholecystectomy in an early stage of the operation. The rate of conversion to open surgery was $25 \%$ in their study. Scott et al. successfully performed laparoscopic cholecystectomy for uncomplicated gall bladder diseases in 20 dogs. Although $30 \%$ of the dogs that underwent cholecystectomy had to be converted to open cholecystectomy, $95 \%$ of the dogs were discharged without reoperation [17]. In these studies, dogs that exhibited abdominal effusion, evidence of gall bladder rupture, persistent elevation of total serum bilirubin concentration, and extrahepatic biliary obstruction on ultrasonography were excluded. These studies concluded that laparoscopic cholecystectomy can be performed successfully in dogs after careful case selection, and may be used as a standard treatment for uncomplicated gall bladder diseases. However, because each of these studies had a small sample size and considered a strictly limited indication for laparoscopic cholecystectomy, the usefulness of laparoscopic cholecystectomy in dogs remained unknown.

We have performed laparoscopic cholecystectomy in all dogs with benign gall bladder diseases since 2008. Our case series included dogs with severe conditions, such as gall bladder rupture, abdominal effusion, or elevation of total serum bilirubin concentration. Therefore, we speculate that the results of the present study reflect the clinical results of laparoscopic cholecystectomy for benign gall bladder diseases more accurately than do those of the previous studies. In our study, the median operative time was $124 \mathrm{~min}$, the conversion rate was $4.1 \%$, and operation-related mortality rate was $5.3 \%$. Regarding the operative time, the median operative time was longer than that reported in previous studies. The reasons for this are as follows. First, our study comprised various cases, ranging from those with no symptoms to those with severe conditions. Particularly difficult cases, as expected, affected the median operative time. Second, we routinely performed a cholangiography, which might also have had an influence on the operative time. Finally, we speculate that the learning curve of this procedure prolonged the operative time. 
A steep learning curve has previously been reported for some laparoscopic surgeries [10, 13, 18, 19]. Several reports have shown that experience and standardization lead to good outcomes, including the shortening of the operative time and improvement of perioperative outcomes. Nonetheless, the median operative time for the last 20 dogs in the present study was satisfactory.

In contrast, we obtained satisfactory results regarding both the conversion rate and perioperative complications. We speculate there might be three reasons for this. First, it seems highly possible that the patients' characteristics resulted in the improvement of the perioperative outcomes. Previous studies have reported that a strict indication for laparoscopic cholecystectomy leads to good results $[12,17]$. However, we obtained excellent results even though we also performed laparoscopic cholecystectomy in dogs with severe conditions. Some reports have shown that dogs with gall bladder mucocele that undergo the operation early have a much better prognosis than do those who do not, because the rate of ruptures is high during the late stages of gall bladder mucocele [1, 16]. From the perspective of laparoscopic surgery, it is more difficult to perform laparoscopic cholecystectomy for a swollen gall bladder, which is often observed at the late stage of gall bladder mucocele and cholecystitis, than for a normal gall bladder, because maintaining an appropriate operative view is difficult $[12,17]$.

We recommended the operation to the owners if their dogs were highly suspected of having gall bladder diseases, such as gall bladder mucocele and cholecystitis, on the basis of imaging, physical, and blood examination results, even if their clinical signs were minor. Further, for dogs that were incidentally diagnosed with benign gall bladder diseases during a medical checkup or observation for other diseases, we recommended the operation to owners if the imaging or blood examination findings of their dogs were exacerbated after waiting, even if they had no clinical symptoms. This indication may result in excellent perioperative results even though we included uncomplicated cases that were excluded in previous studies. Second, the improvement in the procedure led to excellent results. We believe it is extremely difficult to secure the cystic duct first and perform a retrograde dissection from the hepatic fossa, which is similar to the procedure followed for humans, because of the differences in working space and anatomy between dogs and humans. Scott et al. reported that $25 \%$ of cases required conversion to open cholecystectomy even if a strict indication for laparoscopic cholecystectomy was established and experienced veterinarians performed the surgery [17]. This implies that the procedure needs advanced laparoscopic skills and that preoperative diagnoses are quite difficult. In addition, this suggests the need for a special procedure for treating gall bladder diseases in dogs.

Therefore, we started the anterograde laparoscopic cholecystectomy before transecting the cystic duct. Under this procedure, we were able to prevent the misidentification of the cystic duct and injury to the common bile duct and liver. Advanced skills were required when securing the cystic duct in uncomplicated cases owing to an insufficient intraoperative view because of the swelling of the gall bladder caused by the gall bladder mucocele and cholecystitis, resulting in uncontrolled bleeding and injury to the common bile duct. Scott et al. reported that the cases in which the cystic duct could not be secured at the beginning of the operation were converted to open cholecystectomy [17]. We have certainly been able to improve the performance of dissecting the cystic duct by using this procedure. This may have decreased the number of dogs that had to be converted to open cholecystectomy. In addition, we routinely performed a dissection of the layer of subserous tissue of the gall bladder wall. This technique also helped control bleeding from the hepatic fossa during dissections.

Table 5 shows the summary of cases of conversion to open cholecystectomy. Three dogs had to be converted to open cholecystectomy. The reason for conversion was severe intraabdominal adhesion in 2 dogs and insufficient ventilatory impairment related to hyperadrenocorticism under pneumoperitoneum in 1 . We did not continue performing laparoscopic cholecystectomy because we were unable to obtain an appropriate operative view in each of these dogs. However, we believe that it might be possible to perform laparoscopic cholecystectomy if an appropriate operative view is obtained under pneumoperitoneum.

Table 6 shows the summary of 2 cases of intraoperative deaths. One dog died because of acute circulation failure, and the other because of injury to the diaphragm. In the former case, the death could mainly have been caused by the patient's overall poor condition. In the latter case, the death was caused by a technical complication. We speculate that this complication occurred because of the mishandling of an electric scalpel in a narrow space. On the basis of this experience, we have started using an ultrasonic incision coagulating device instead of an electric scalpel, and have thereafter had no similar complications to date. The intraoperative death occurred in each case at the time of initiation of laparoscopic cholecystectomy. We have not observed intraoperative death in the later cases included in this series.

This study has some limitations. This was a retrospective study, and the information was retrieved from medical records. Further, all the data used in the present study were retrieved from the operations performed by one veterinarian in one hospital, which therefore, resulted in a lack of diversity. In the future, we intend to analyze and compare further studies on open cholecystectomy.

In summary, we report the short-term results of laparoscopic cholecystectomy for benign gall bladder diseases in dogs. It is a feasible, safe, and appropriate procedure considering the operative indications.

ACKNOWLEDGMENTS. We would like to thank Dr. Motoo Yamagata, Dr. Minoru Matsuda, and Mr. Mark Richley for giving us the opportunity to discuss the procedure of laparoscopic cholecystectomy in dogs.

\section{REFERENCES}

1. Aguirre, A. L., Center, S. A., Randolph, J. F., Yeager, A. E., Keegan, A. M., Harvey, H. J. and Erb, H. N. 2007. Gallbladder disease in Shetland Sheepdogs: 38 cases (1995-2005). J. Am. Vet. Med. Assoc. 231: 79-88. [Medline] [CrossRef]

2. Amsellem, P. M., Seim, H. B. 3rd., MacPhail, C. M., Bright, R. M., Twedt, D. C., Wrigley, R. H. and Monnet, E. 2006. Long-term survival and risk factors associated with biliary surgery in dogs: 34 cases (1994-2004). J. Am. Vet. Med. Assoc. 229: 1451-1457. [Medline] [CrossRef] 
3. Arulpragasam, S. P., Case, J. B. and Ellison, G. W. 2013. Evaluation of costs and time required for laparoscopic-assisted versus open cystotomy for urinary cystolith removal in dogs: 43 cases (2009-2012). J. Am. Vet. Med. Assoc. 243: 703-708. [Medline] [CrossRef]

4. Barkun, J. S., Barkun, A. N., Sampalis, J. S., Fried, G., Taylor, B., Wexler, M. J., Goresky, C. A. and Meakins, J. L. 1992. Randomised controlled trial of laparoscopic versus mini cholecystectomy. The McGill Gallstone Treatment Group. Lancet 340: 1116-1119. [Medline] [CrossRef]

5. Case, J. B., Boscan, P. L., Monnet, E. L., Niyom, S. R., Imhoff, D. J., Wallace, M. L. and Smeak, D. D. 2015. Comparison of surgical variables and pain in cats undergoing ovariohysterectomy, laparoscopic-assisted ovariohysterectomy, and laparoscopic ovariectomy. J. Am. Anim. Hosp. Assoc. 51: 1-7. [Medline] [CrossRef]

6. Crews, L. J., Feeney, D. A., Jessen, C. R., Rose, N. D. and Matise, I. 2009. Clinical, ultrasonographic, and laboratory findings associated with gallbladder disease and rupture in dogs: 45 cases (1997-2007). J. Am. Vet. Med. Assoc. 234: 359-366. [Medline] [CrossRef]

7. Culp, W. T., Mayhew, P. D. and Brown, D. C. 2009. The effect of laparoscopic versus open ovariectomy on postsurgical activity in small dogs. Vet. Surg. 38: 811-817. [Medline] [CrossRef]

8. Dindo, D., Demartines, N. and Clavien, P. A. 2004. Classification of surgical complications: a new proposal with evaluation in a cohort of 6336 patients and results of a survey. Ann. Surg. 240: 205-213. [Medline] [CrossRef]

9. Hendolin, H. I., Pääkönen, M. E., Alhava, E. M., Tarvainen, R., Kemppinen, T. and Lahtinen, P. 2000. Laparoscopic or open cholecystectomy: a prospective randomised trial to compare postoperative pain, pulmonary function, and stress response. Eur. J. Surg. 166: 394-399. [Medline] [CrossRef]

10. Jung, D. H., Son, S. Y., Park, Y. S., Shin, D. J., Ahn, H. S., Ahn, S. H., Park, D. J. and Kim, H. H. 2016. The learning curve associated with laparoscopic total gastrectomy. Gastric Cancer 19: 264-272. [Medline] [CrossRef]

11. Malek, S., Sinclair, E., Hosgood, G., Moens, N. M., Baily, T. and Boston, S. E. 2013. Clinical findings and prognostic factors for dogs undergoing cholecystectomy for gall bladder mucocele. Vet. Surg. 42: 418-426. [Medline] [CrossRef]

12. Mayhew, P. D., Mehler, S. J. and Radhakrishnan, A. 2008. Laparoscopic cholecystectomy for management of uncomplicated gall bladder mucocele in six dogs. Vet. Surg. 37: 625-630. [Medline] [CrossRef]

13. Mayhew, P. D., Culp, W. T., Hunt, G. B., Steffey, M. A., Mayhew, K. N., Fuller, M., Della-Maggiore, A. and Nelson, R. W. 2014. Comparison of perioperative morbidity and mortality rates in dogs with noninvasive adrenocortical masses undergoing laparoscopic versus open adrenalectomy. $J$. Am. Vet. Med. Assoc. 245: 1028-1035. [Medline] [CrossRef]

14. Mehler, S. J., Mayhew, P. D., Drobatz, K. J. and Holt, D. E. 2004. Variables associated with outcome in dogs undergoing extrahepatic biliary surgery: 60 cases (1988-2002). Vet. Surg. 33: 644-649. [Medline] [CrossRef]

15. NIH Consensus conference. 1993. Gallstones and laparoscopic cholecystectomy. JAMA 269: 1018-1024. [Medline] [CrossRef]

16. Pike, F. S., Berg, J., King, N. W., Penninck, D. G. and Webster, C. R. 2004. Gallbladder mucocele in dogs: 30 cases (2000-2002). J. Am. Vet. Med. Assoc. 224: 1615-1622. [Medline] [CrossRef]

17. Scott, J., Singh, A., Mayhew, P. D., Brad Case, J., Runge, J. J., Gatineau, M. and Kilkenny, J. 2016. Perioperative complications and outcome of laparoscopic cholecystectomy in 20 dogs. Vet. Surg. 45 S1: O49-O59. [Medline] [CrossRef]

18. Tekkis, P. P., Senagore, A. J., Delaney, C. P. and Fazio, V. W. 2005. Evaluation of the learning curve in laparoscopic colorectal surgery: comparison of right-sided and left-sided resections. Ann. Surg. 242: 83-91. [Medline] [CrossRef]

19. Victorzon, M., Peromaa-Haavisto, P. and Tolonen, P. 2012. Perioperative morbidity, mortality and early outcome of the first 360 gastric bypass operations performed in a district hospital. Scand. J. Surg. 101: 184-189. [Medline] [CrossRef]

20. Worley, D. R., Hottinger, H. A. and Lawrence, H. J. 2004. Surgical management of gallbladder mucoceles in dogs: 22 cases (1999-2003). J. Am. Vet. Med. Assoc. 225: 1418-1422. [Medline] [CrossRef] 